\title{
Correction to: J Med Food 2016;19(6):569-577
}

In the June issue of Journal of Medicinal Food (vol. 19, no. 6; 569-577), the article "Aqueous Extraction of Citrus unshiu Peel Induces Proangiogenic Effects Through the FAK and ERK1/2 Signaling Pathway in Human Umbilical Vein Endothelial Cells" by Lee et al. requires correction. It has come to our attention that co-author Il-woung Kim's name was misspelled as Il-woong Kim.

The online version of the article has been corrected to reflect these changes.

The authors apologize for this error. 